\title{
Reproducibility of the ambulatory arterial stiffness index in hypertensive patients
}

\author{
Dirk G. Dechering ${ }^{\mathrm{a}, \mathrm{b}}$, Marijke S. van der Steen ${ }^{\mathrm{a}}$, Ahmet Adiyaman ${ }^{\mathrm{a}}$, \\ Lutgarde Thijs $^{b}$, Jaap Deinum ${ }^{\mathrm{a}}$, Yan $\mathrm{Li}^{\mathrm{c}}$, Eamon Dolan ${ }^{\mathrm{d}}$, \\ Reinier P.M. Akkermans ${ }^{a}$, Tom Richart ${ }^{\text {b,e }}$, Tine W. Hansen ${ }^{f}$, Masahiro Kikuya ${ }^{g}$, \\ Jiguang Wang ${ }^{c}$, Eoin O'Brien ${ }^{\mathrm{h}}$, Theo Thien ${ }^{\mathrm{a}}$ and Jan A. Staessen ${ }^{\mathrm{b}, \mathrm{e}}$
}

Background We studied the repeatability of the ambulatory arterial stiffness index (AASI), which can be computed from 24-h blood pressure (BP) recordings as unity minus the regression slope of diastolic on systolic BP.

Methods One hundred and fifty-two hypertensive outpatients recruited in Nijmegen (mean age $=46.2$ years; 76.3\% with systolic and diastolic hypertension) and 145 patients enrolled in the Systolic Hypertension in Europe (Syst-Eur) trial (71.0 years) underwent 24-h BP monitoring at a median interval of 8 and 31 days, respectively. We used the repeatability coefficient, which is twice the SD of the within-participant differences between repeat recordings, and expressed it as a percentage of four times the SD of the mean of the paired measurements.

Results Mean AASI (crude or derived by time-weighted or robust regression) and 24-h pulse pressure (PP) were similar on repeat recordings in both cohorts. In Nijmegen patients, repeatability coefficients of AASI and PP were $\sim 50 \%$. In Syst-Eur trial patients, repeatability coefficient was $\sim 60 \%$ for AASI and $\sim \mathbf{4 0} \%$ for PP. For comparison, repeatability coefficients for 24-h systolic and diastolic BP were $\sim 30 \%$. Differences in AASI between paired recordings were correlated with differences in the goodness of fit $\left(r^{2}\right)$ of the AASI regression line as well as with differences in the night-to-day BP ratio. However, in sensitivity analyses stratified for type of hypertension, $r^{2}$, or dipping status, repeatability coefficients for AASI did not widely depart from 50 to $60 \%$ range.

\section{Introduction}

Increased arterial stiffness of the large arteries predicts cardiovascular complications over and beyond blood pressure (BP) and classical cardiovascular risk factors [1]. Nevertheless, risk stratification based on arterial stiffness remains underused in clinical practice because most techniques to measure arterial properties require expensive equipment and highly trained observers. We recently proposed the ambulatory arterial stiffness index (AASI) as a measure reflecting arterial stiffness [2,3]. This novel index, defined as one minus the regression slope of diastolic on systolic BP in individual subjects, can be determined from 24-h ambulatory BP recordings [2,3].
Conclusion Estimates of mean AASI were not different between repeat recordings, and repeatability coefficients were within the 50-60\% range. J Hypertens 26:1993-2000 (C) 2008 Wolters Kluwer Health | Lippincott Williams \& Wilkins.

Journal of Hypertension 2008, 26:1993-2000

Keywords: ambulatory arterial stiffness index, ambulatory blood pressure monitoring, arterial stiffness, diurnal blood pressure profile, reproducibility

Abbreviations: AASI, ambulatory arterial stiffness index; baPWV, brachialankle pulse wave velocity; cAIX, carotid augmentation index; IDACO, International Database on Ambulatory BP monitoring in relation to Cardiovascular Outcomes; IQR, interquartile range; QKD, QRS-Korotkoff delay; SDD, standard deviation of differences; Syst-Eur, SYSTolic hypertension in EURope trial

aDepartment of General Internal Medicine, University Medical Centre St Radboud, Radboud University, Nijmegen, The Netherlands, ' ${ }^{\text {S Studies }}$

Coordinating Centre, Division of Hypertension and Cardiovascular Rehabilitation, Department of Cardiovascular Diseases, University of Leuven, Leuven, Belgium, ${ }^{\mathrm{C}}$ Center for Epidemiological Studies and Clinical Trials, Ruijin Hospital, Shanghai Jiaotong University School of Medicine, Shanghai, China, ${ }^{d}$ Cambridge University Hospitals, Addenbrook's Hospital, Cambridge, UK, ' Genetic Epidemiology Unit, Department of Epidemiology, Maastricht University, The Netherlands, 'Research Center for Prevention and Health, and Department of Clinical Physiology, Hvidovre University Hospital, Copenhagen, Denmark, ${ }^{9}$ Tohoku University Graduate School of Pharmaceutical Sciences and Medicine, Sendai, Japan and ${ }^{\mathrm{h}}$ Conway Institute of Biomolecular and Biomedical Research, University College Dublin, Dublin, Ireland

Correspondence to Jan Staessen, Division of Hypertension and Cardiovascular Rehabilitation, Department of Cardiovascular Diseases, University of Leuven, Campus Gasthuisberg, Herestraat 49, Box 702, B-3000 Leuven, Belgium Tel: +32 1634 7104; fax: +32 16347106 ;

e-mail: jan.staessen@med.kuleuven.be

Received 14 February 2008 Revised 7 May 2008 Accepted 23 May 2008

To date, several cross-sectional studies $[4,5]$ and at least three prospective cohort studies $[3,6,7]$ demonstrated an association of AASI either with signs of target organ damage in never-treated hypertensive patients [4] or with the incidence of cardiovascular mortality and morbidity $[3,6,7]$. When adjusted for pulse pressure (PP) $[3,6,7]$ or aortic pulse wave velocity [8], AASI remained predictive, in particular of stroke.

To our knowledge, no previous publication has addressed the reproducibility of AASI as determined from repeat ambulatory BP recordings. We investigated the reproducibility of AASI in patients with systolic and/or diastolic 
hypertension recruited in Nijmegen and in older patients with isolated systolic hypertension enrolled in a substudy to the Systolic Hypertension in Europe (Syst-Eur) trial.

\section{Methods \\ Study population}

Patients referred to the outpatient clinic of the University Medical Centre St Radboud, Nijmegen, the Netherlands [9], and patients enrolled in the substudy on ambulatory BP measurement to the Syst-Eur trial [10] qualified for the present analysis, if they had undergone repeat 24-h ambulatory recordings within 2 months, while being untreated $[9,10]$ or minimally treated [9] with no change in treatment status between the first and repeat recording.

\section{Nijmegen cohort}

At the Nijmegen outpatient clinic, doctors discontinued BP-lowering drugs in newly referred patients, except when there was a compelling indication, such as the use of $\beta$-blockers in patients with angina pectoris ('minimal treatment'). The office BP was then reassessed by obtaining three consecutive measurements at two to three follow-up visits. Of 1325 consecutive patients attending the Nijmegen clinic, 275 underwent repeat ambulatory BP measurement within 2 months. Of those, we excluded $33(12.0 \%)$ from analysis because their treatment status changed between the first and repeat recording, and $56(20.4 \%)$ because over the whole day the mean interval between the ambulatory BP readings was shorter than $15 \mathrm{~min}$ or longer than $45 \mathrm{~min}$.

\section{The Systolic Hypertension in Europe trial cohort}

Previous publications describe the protocol of the SystEur trial in detail [10]. Eligible patients were aged 60 years or older. They had a sitting BP ranging from 160 to $219 \mathrm{mmHg}$ systolic and less than $95 \mathrm{mmHg}$ diastolic. The standing systolic BP had to be at least $140 \mathrm{mmHg}$. Of 837 patients [10] enrolled in the substudy on ambulatory BP monitoring, 153 (18.3\%) had repeat recordings within 2 months, while taking placebo.

\section{Blood pressure measurement}

Trained observers measured the patients' office BP at repeated visits. To describe the patients' conventional $\mathrm{BP}$, we used the average of six to nine readings (three readings at two to three visits) in the Nijmegen cohort [9] and the average of six readings (two readings at three visits) in Syst-Eur trial patients [10]. For the definition of hypertension in the sensitivity analyses, we applied current guidelines [11]. Isolated systolic hypertension was a BP of $140 \mathrm{mmHg}$ systolic or higher with diastolic BP below $90 \mathrm{mmHg}$.

For analysis, we considered ambulatory BP recordings with the interval between successive readings programmed at intervals from 15 to $30 \mathrm{~min}$ during daytime and from 30 to $60 \mathrm{~min}$ during nighttime. We only analysed recordings with at least five readings between midnight and 06:00 $\mathrm{h}$. In addition, we required that the recordings included at least 15 daytime readings. On the basis of these quality criteria, we excluded 34 patients from the Nijmegen cohort (12.4\%) and eight Syst-Eur trial patients (5.2\%). Thus, the number of patients analysed totalled 297, that is 152 patients from Nijmegen and 145 Syst-Eur trial patients.

The validated monitors used in Nijmegen were the auscultatory Oxford Medilog (Oxford Medical Systems Ltd, Oxford, UK [12]) in 54 patients (35.5\%), the oscillometric Mobil O Graph (I.E.M., Stolberg, Germany [13]) in 43 patients (28.3\%), and the oscillometric SpaceLabs 90207 (SpaceLabs Inc., Redmond, Washington, USA [14]) in 55 patients $(36.2 \%)$. The monitors fitted to Syst-Eur trial patients were the oscillometric SpaceLabs 90202 [14] or 90207 [15] in 49 patients $(35.5 \%)$ and 78 patients $(56.5 \%)$, respectively, and the auscultatory Takeda TM2420 (A\&D, Tokyo, Japan [16]) in 11 patients $(3.7 \%)$.

The same SAS macro processed all ambulatory recordings, which stayed unedited. For the sensitivity analysis involving dipping status, we defined daytime and nighttime in both cohorts as the intervals ranging from 10:00 to $20: 00 \mathrm{~h}$ and from midnight to $06: 00 \mathrm{~h}$, respectively $[17,18]$. We weighted the individual means of the ambulatory $\mathrm{BP}$ by the interval between readings.

From individual 24-h recordings, we computed the regression slope of diastolic on systolic BP. We defined the AASI as one minus the regression slope (crude AASI). We used the coefficient of determination $\left(r^{2}\right)$ as a measure of the goodness of fit of the AASI regression line. We also computed AASI, using time-weighted or robust regression (least trimmed squares).

\section{Statistical methods}

For database management and statistical analysis, we used SAS software (version 9.1.3; SAS Institute, Cary, North Carolina, USA). For comparison of means, we applied the Student's $t$-test for paired or unpaired observations, as appropriate. For comparison of proportions between groups, we applied the $\chi^{2}$-statistic. Statistical significance was an $\alpha$-level of 0.05 on two-sided tests.

We assessed the agreement between paired ambulatory recordings by Bland and Altman's approach $[19,20]$. For the evaluation of reproducibility, we used the repeatability coefficient, which is twice the SD of the withinparticipant differences between repeat recordings (repeat minus first) [21]. This measure is specifically designed to evaluate within-participant reproducibility. To take into consideration the normal biological variation of a measure, we expressed the repeatability coefficient as a percentage of close to maximal variation (four times the 
Table 1 Characteristics of patients at first ambulatory recording

\begin{tabular}{lccc}
\hline Characteristic & Nijmegen & Syst-Eur & $P$ \\
\hline Number of participants & 152 & 145 & \\
Mean (SD) of characteristic & & & \\
Age (years) & $46.2 \pm 13.6$ & $71.0 \pm 6.5$ & $<0.0001$ \\
Weight (kg) & $77.9 \pm 15.3$ & $69.4 \pm 13.6$ & $<0.0001$ \\
Height (cm) & $170 \pm 9$ & $162 \pm 10$ & $<0.0001$ \\
Body mass index (kg/m $\left.{ }^{2}\right)$ & $26.8 \pm 4.7$ & $26.4 \pm 4.2$ & 0.46 \\
Office systolic BP (mmHg) & $168.6 \pm 25.1$ & $178.4 \pm 16.6$ & $<0.0001$ \\
Office diastolic BP (mmHg) & $101.2 \pm 11.4$ & $85.4 \pm 9.0$ & $<0.0001$ \\
Pulse rate (beats per minute) & $72.9 \pm 12.5$ & $73.5 \pm 11.1$ & 0.35 \\
Number of participants (\%) with characteristic & & \\
Women, $n$ (\%) & $89(58.6)$ & $89(61.4)$ & 0.62 \\
Smokers, $n$ (\%) & $21(14.2)$ & $15(10.3)$ & 0.32 \\
Drinking alcohol, $n(\%)$ & $22(14.5)$ & $34(23.8)$ & 0.05 \\
On BP lowering drugs, $n$ (\%) & $18(11.8)$ & $0(0.0)$ & $<0.0001$ \\
Diabetes mellitus, $n(\%)$ & $11(7.2)$ & $11(7.6)$ & 0.99 \\
Classification of hypertension ${ }^{\mathrm{a}}$ & & & \\
Isolated systolic, $n(\%)$ & $17(11.2)$ & $100(69.0)$ & $<0.0001$ \\
Isolated diastolic, $n(\%)$ & $12(7.9)$ & $0(0.0)$ & 0.001 \\
Mixed, $n$ (\%) & $116(76.3)$ & $44(30.3)$ & $<0.0001$ \\
History of cardiovascular disease, $n(\%)$ & $22(14.5)$ & $44(30.3)$ & 0.001 \\
\hline
\end{tabular}

$P$ values are for the differences between the patients enrolled in the Nijmegen cohort and in the Syst-Eur trial. AASI, ambulatory arterial stiffness index. ${ }^{a}$ A total of seven patients in Nijmegen and one from the Systolic Hypertension in Europe (Syst-Eur) trial were not hypertensive on clinic blood pressure (BP) measurement when taking into account current hypertension guidelines.

SD of the mean of the duplicate measurements). By this, a reasonable comparison of the repeatability of different measures can be undertaken.

We also applied single and multiple regression analysis. In stepwise regression, we set the $P$-value for independent variables to enter and to stay into models at 0.15 . We studied the coincidence of regression lines by analysis of covariance, as described by Kleinbaum et al. [22].

\section{Results}

\section{Patient characteristics}

Table 1 lists the main clinical characteristics of the two study cohorts. Both groups of patients included nearly $60 \%$ of women. Compared with Syst-Eur trial patients, the Nijmegen patients were younger ( 46.2 versus 71.0 years), taller $(170$ versus $162 \mathrm{~cm})$, heavier $(77.9$ versus $69.4 \mathrm{~kg}$ ), but had similar body mass index (26.8 versus $\left.26.4 \mathrm{~kg} / \mathrm{m}^{2}\right)$.

In line with the recruitment criteria based on office BP, Syst-Eur compared with Nijmegen patients (Table 1) had higher systolic BP (178.4 versus $168.6 \mathrm{mmHg}$ ) on conventional measurement, but lower diastolic BP (85.4 versus $101.2 \mathrm{mmHg}$ ). A history of cardiovascular disease was more prevalent among the older Syst-Eur trial patients (30.3 versus $14.5 \%)$. Table 2 shows that SystEur trial patients on average had higher AASI than Nijmegen patients, irrespective of the way AASI was computed (all $P<0.008$ ).

\section{Repeatability of ambulatory measurements}

Figure 1 provides Bland and Altman plots for the crude AASI in Nijmegen and Syst-Eur trial patients, respectively. Detailed repeatability statistics appear in Table 2.

\section{Nijmegen cohort}

The median interval between the first and repeat ambulatory recording was 8 days [range $=4-60$ days; interquartile range $(\mathrm{IQR})=7-15$ days]. AASI, time-weighted AASI, robust AASI and 24-h PP were similar on first and repeat measurement ( $P \geq 0.10$; Table 2). The 24-h diastolic BP was significantly lower on repeat than first recording $(-1.43 \mathrm{mmHg} ; P=0.009)$ with a similar trend for the 24-h systolic BP $(-1.51 \mathrm{mmHg} ; P=0.09)$. The repeatability of AASI and 24-h PP expressed as a percentage of the near maximal variation in these measurements (the interval corresponding with $\pm 2 \mathrm{SD}$ around the mean of the repeat recordings) ranged from approximately 45 to $55 \%$. The corresponding repeatability estimate for the 24-h systolic and diastolic BP was around 30\% (Table 2).

In Nijmegen patients, the differences between repeat minus first crude AASI correlated inversely with the differences in the goodness of fit of the AASI regression

Table 2 Differences between repeat minus first ambulatory measurement

\begin{tabular}{|c|c|c|c|c|c|c|c|}
\hline & First & Repeat & $P$ & $\Delta$ Absolute & $95 \% \mathrm{Cl}$ & $\mathrm{RC}$ & pMV (\%) \\
\hline \multicolumn{8}{|l|}{ Nijmegen $(n=152)$} \\
\hline Crude AASI & $0.47 \pm 0.16$ & $0.45 \pm 0.17$ & 0.13 & $-0.02 \pm 0.16$ & -0.04 to 0.01 & 0.32 & 55 \\
\hline Time-weighted AASI & $0.47 \pm 0.16$ & $0.43 \pm 0.17$ & 0.10 & $-0.02 \pm 0.16$ & -0.05 to 0.01 & 0.32 & 55 \\
\hline Robust AASI & $0.43 \pm 0.17$ & $0.42 \pm 0.19$ & 0.28 & $-0.01 \pm 0.15$ & -0.04 to 0.01 & 0.30 & 46 \\
\hline 24-h systolic BP ( $\mathrm{mmHg})$ & $150.1 \pm 19.2$ & $148.6 \pm 18.4$ & 0.09 & $-1.51 \pm 10.7$ & -3.23 to 0.21 & 21.4 & 30 \\
\hline 24-h diastolic BP $(\mathrm{mmHg})$ & $94.2 \pm 13.1$ & $92.7 \pm 12.6$ & 0.009 & $-1.43 \pm 6.69$ & -2.50 to -0.36 & 13.4 & 27 \\
\hline 24-h PP (mmHg) & $56.0 \pm 12.1$ & $55.9 \pm 12.5$ & 0.88 & $-0.08 \pm 7.00$ & -1.15 to 0.99 & 14.0 & 52 \\
\hline \multicolumn{8}{|l|}{ Syst-Eur trial $(n=145)$} \\
\hline Crude AASI & $0.52 \pm 0.19$ & $0.51 \pm 0.20$ & 0.40 & $-0.02 \pm 0.20$ & -0.05 to 0.02 & 0.40 & 61 \\
\hline Time-weighted AASI & $0.52 \pm 0.20$ & $0.51 \pm 0.20$ & 0.29 & $-0.02 \pm 0.20$ & -0.05 to 0.02 & 0.40 & 61 \\
\hline Robust AASI & $0.55 \pm 0.16$ & $0.54 \pm 0.15$ & 0.66 & $-0.01 \pm 0.16$ & -0.03 to 0.02 & 0.32 & 62 \\
\hline 24-h systolic BP (mmHg) & $154.7 \pm 15.4$ & $153.4 \pm 15.7$ & 0.09 & $-1.30 \pm 9.11$ & -2.79 to 0.20 & 18.2 & 31 \\
\hline 24-h diastolic BP $(\mathrm{mmHg})$ & $81.1 \pm 9.7$ & $80.4 \pm 8.9$ & 0.12 & $-0.71 \pm 5.95$ & -1.68 to 0.19 & 11.9 & 33 \\
\hline 24-h PP (mmHg) & $73.6 \pm 12.2$ & $73.0 \pm 12.9$ & 0.24 & $-1.45 \pm 9.21$ & -1.71 to 0.32 & 18.4 & 37 \\
\hline
\end{tabular}

Plus-minus values are mean $\pm \mathrm{SD}$. Absolute differences $(\Delta)$ were computed as repeat minus first recording. The repeatability coefficient (RC) is twice the $\mathrm{SD}$ of the signed differences between the duplicate measurements. RC was also expressed as a percentage of four times the SD of the mean of the paired recordings (pMV). AASI, ambulatory arterial stiffness index; BP, blood pressure; Cl, confidence interval; PP, pulse pressure; Syst-Eur, Systolic Hypertension in Europe. 
Fig. 1
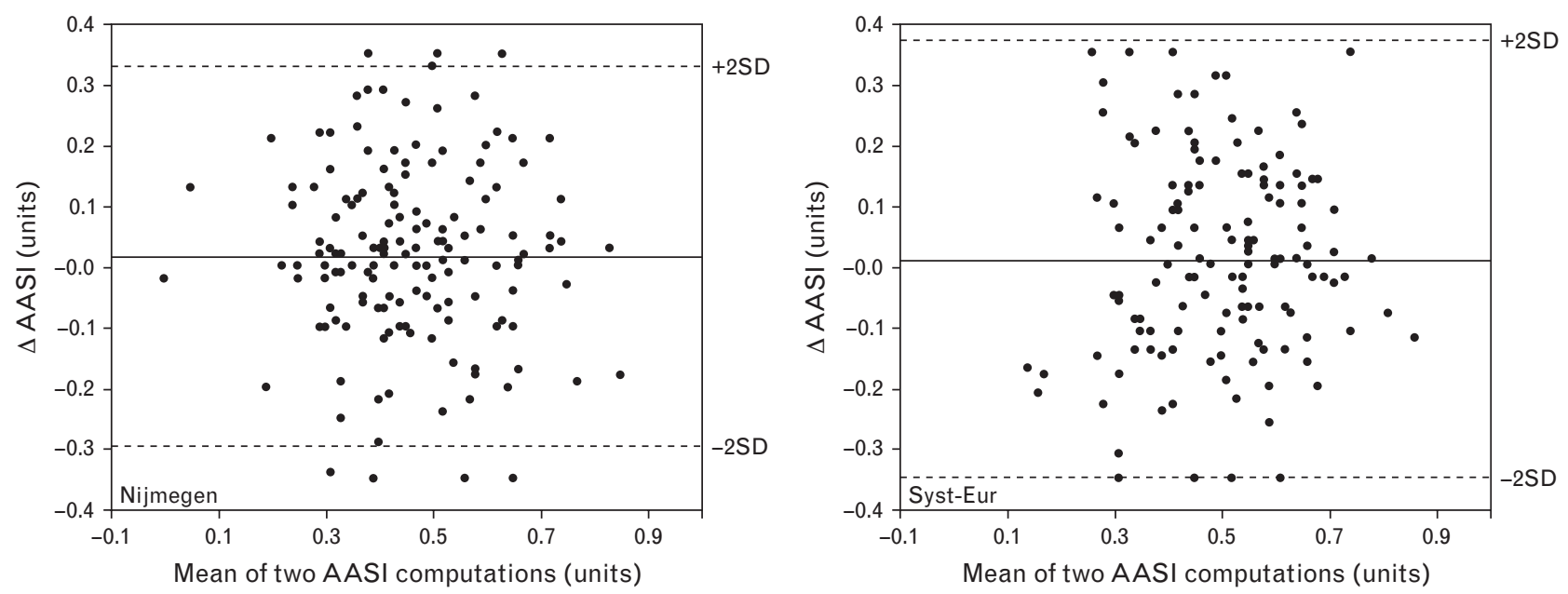

Reproducibility of the ambulatory arterial stiffness index (crude AASI) in patients enrolled in the Nijmegen and Syst-Eur trial cohorts. The difference of the second minus the first measurement of AASI was plotted against the mean of the repeat measurements (Bland-Altman plot). The full and dotted lines represent the mean difference and the limits of agreement (mean \pm 2 SD), respectively. Outliers were plotted at \pm 0.35 . AASI, arterial stiffness index.

line $(r=-0.77 ; P<0.0001)$ and the changes in the nightto-day ratios of diastolic BP $(r=-0.29 ; P=0.0003)$, but not systolic BP $(r=-0.12 ; \quad P=0.14)$. In multiple regression analyses, with mutual adjustments for these covariates, the corresponding partial correlation coefficients were $-0.76(P<0.0001),-0.25(P=0.003)$, and $-0.25(P=0.002)$, respectively.

\section{The Systolic Hypertension in Europe trial patients}

The median interval between the first and repeat ambulatory recording was 31 days (range $=2-57$ days; $\mathrm{IQR}=28-35$ days). AASI, time-weighted AASI, robust AASI, 24-h PP and 24-h systolic and diastolic BP were similar on first and repeat measurement $(P \geq 0.09$; Table 2). Estimates of repeatability expressed as a percentage of near maximal variation were close to $60 \%$ for AASI, around $40 \%$ for the $24-\mathrm{h} \mathrm{PP}$, and approximately $30 \%$ for 24-h systolic and diastolic BP (Table 2).

In Syst-Eur trial patients, the differences between repeat minus first crude AASI correlated inversely with the differences in the goodness of fit of the AASI regression line $(r=-0.57 ; P<0.0001)$ and those in the night-to-day ratios of diastolic $\mathrm{BP}(r=-0.25 ; P=0.003)$ with a similar trend for the differences in the night-to-day ratio of systolic BP $(r=-0.15 ; P=0.07)$. The corresponding partial correlation coefficients with mutual adjustment for these covariates were $-0.55(P<0.0001),-0.30$ $(P=0.001)$, and $-0.23(P=0.006)$, respectively.

\section{Comparison of repeatability between Nijmegen and the Systolic Hypertension in Europe trial patients}

None of the differences in the estimates of repeatability among paired recordings between Nijmegen and Syst-
Eur trial patients reached significance for any of the variables listed in Table $2(P \geq 0.10)$. For the sensitivity analyses, we therefore pooled Nijmegen and Syst-Eur trial patients.

The regression lines for the differences among the paired recordings in crude AASI versus those in the goodness of fit of the AASI regression lines were not coincident in Nijmegen and Syst-Eur trial patients because the slope was steeper in the Nijmegen patients $(P=0.008$; Fig. 2$)$. In contrast, the regression lines for the differences among the paired recordings in crude AASI versus those in the night-to-day BP ratio were coincident ( $P \geq 0.70$; Fig. 3).

\section{Sensitivity analyses}

Table 3 shows sensitivity analyses, according to the type of hypertension, dipping status, and the $r^{2}$ of the AASI regression line. In these analyses, we defined nondipping as a diastolic night-to-day BP ratio higher than 0.90 . For the goodness of fit of the AASI regression line, we used 0.36 as the cut-off threshold, because the association of AASI with four determinants of arterial stiffness increased curvilinearly with $r^{2}$, with most of the improvement in the association occurring above a value of $r^{2}$ of 0.36 .

\section{Discussion}

The key finding of our study was that AASI as determined from ambulatory BP recordings repeated within an interval of 2 months had moderate reproducibility. Mean values of AASI were similar in paired recordings. The repeatability coefficient, which is a measure for reproducibility within individual participants, was within the $50-60 \%$ range, which is better than for other parameters derived from the diurnal BP profile [23]. For comparison, 


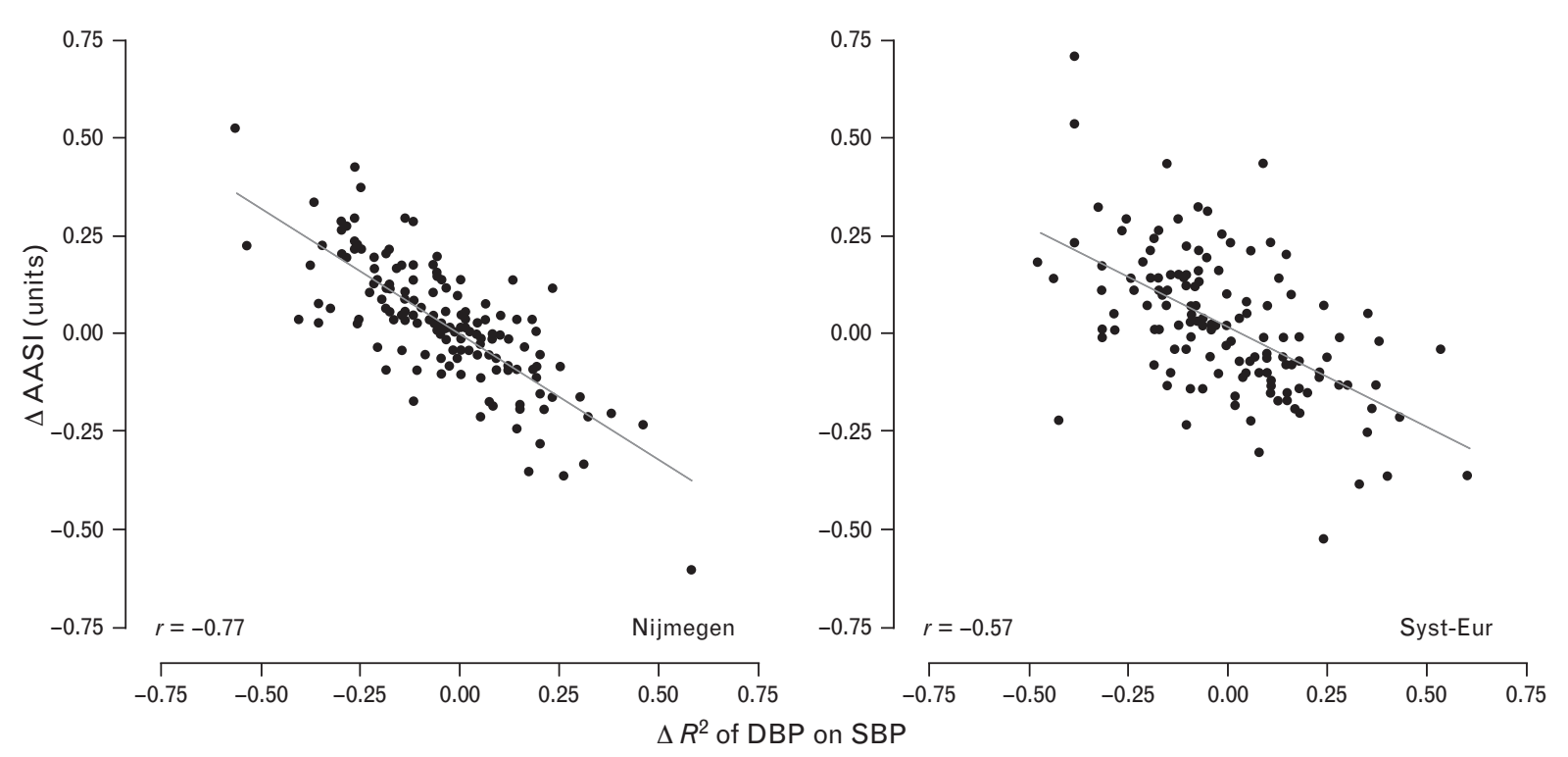

Association between the differences (repeat minus first) between repeat measurements of the ambulatory arterial stiffness index (crude AASI) and the differences (repeat minus first) in the goodness of fit of the AASI regression lines $\left(r^{2}\right)$ in individual patients enrolled in the Nijmegen and Syst-Eur trial cohorts, respectively. The slope was significantly steeper in Nijmegen than Syst-Eur trial patients $(P=0.008)$. DBP, diastolic blood pressure; SBP, systolic blood pressure.

the repeatability coefficients of the 24-h systolic and diastolic BPs in the current study were close to $30 \%$, but diastolic BP in the Nijmegen cohort showed significant regression to the mean with lower values of the 24-h diastolic BP on repeat recording.
$\mathrm{PP}$, the QRS-Korotkoff-delay (QKD) index standardized to a systolic BP of $100 \mathrm{mmHg}$ and a heart rate of 60 beats per minute (QKD100-60), the systolic augmentation index and pulse wave velocity are other noninvasive measures of arterial stiffness. Stergiou et al. [24] investi-

\section{Fig. 3}

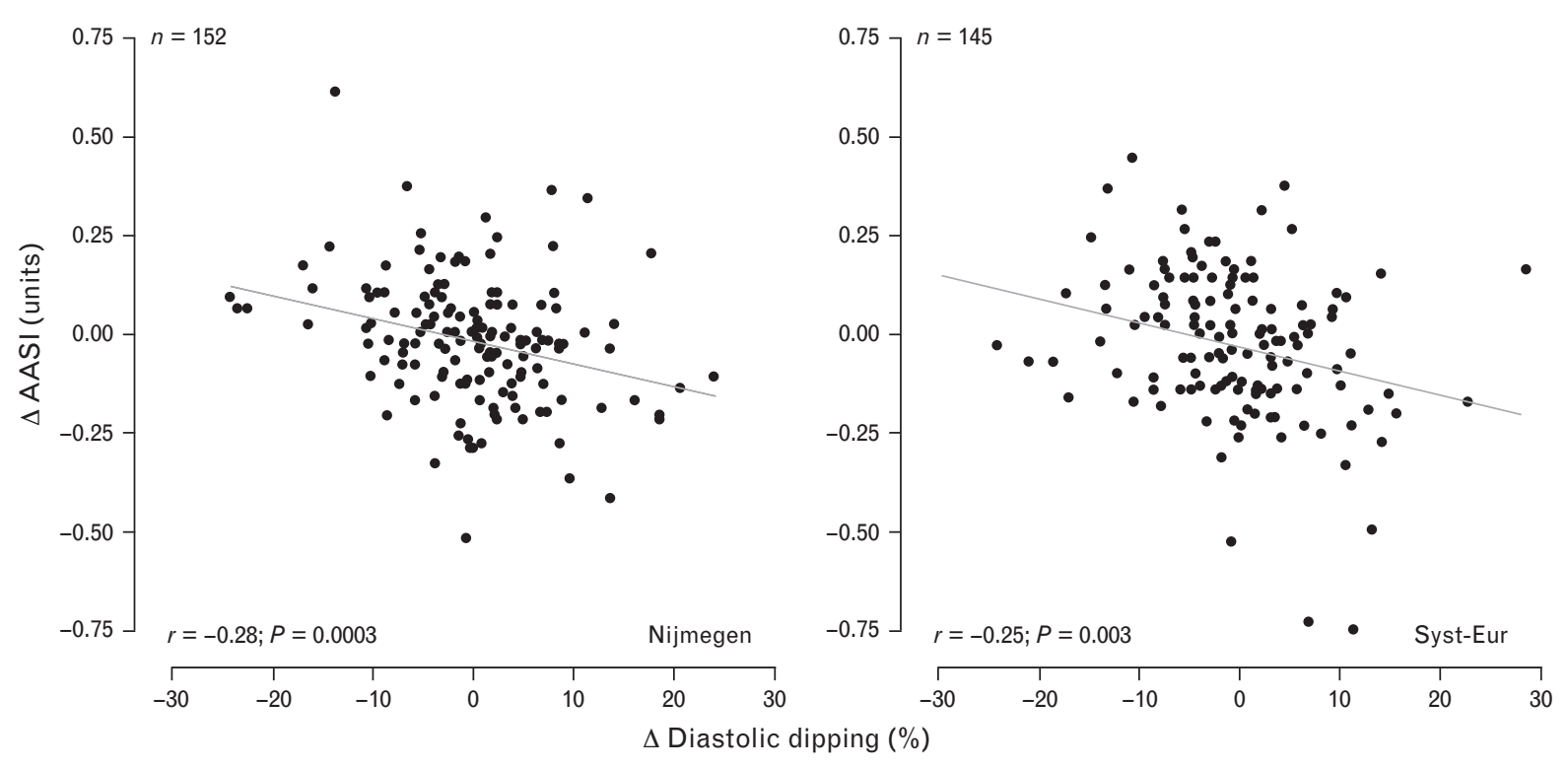

Association between the differences between repeat measurements of the ambulatory arterial stiffness index (crude AASI) and the differences in the night-do-day ratio of diastolic blood pressure in individual patients enrolled in the Nijmegen and Syst-Eur cohorts, respectively. The regression lines in Nijmegen and Syst-Eur trial patients were coincident. 
Table 3 Differences between repeat minus first ambulatory measurement in subgroups

\begin{tabular}{|c|c|c|c|c|c|c|c|}
\hline & First & Repeat & $P$ & $\Delta$ Absolute & $95 \% \mathrm{Cl}$ & $\mathrm{RC}$ & pMV (\%) \\
\hline \multicolumn{8}{|l|}{ Type of hypertension } \\
\hline Isolated systolic $(n=117)$ & $0.54 \pm 0.19$ & $0.51 \pm 0.19$ & 0.09 & $-0.03 \pm 0.19$ & -0.06 to 0.01 & 0.38 & 64 \\
\hline Isolated diastolic $(n=12)$ & $0.36 \pm 0.16$ & $0.43 \pm 0.17$ & 0.09 & $0.07 \pm 0.13$ & -0.01 to 0.15 & 0.26 & 43 \\
\hline Mixed systolic and diastolic $(n=160)$ & $0.48 \pm 0.16$ & $0.46 \pm 0.18$ & 0.25 & $-0.02 \pm 0.17$ & -0.04 to 0.01 & 0.34 & 51 \\
\hline \multicolumn{8}{|l|}{ Dipping status } \\
\hline Consistent dipper $(n=198)$ & $0.46 \pm 0.17$ & $0.45 \pm 0.13$ & 0.18 & $-0.02 \pm 0.17$ & -0.04 to 0.01 & 0.34 & 57 \\
\hline $\begin{array}{l}\text { Consistent nondipper }(n=31) \\
\text { Inconsistent between recordings }(n=\end{array}$ & $0.61 \pm 0.15$ & $0.60 \pm 0.22$ & 0.88 & $-0.01 \pm 0.21$ & $-0.08 \pm 0.07$ & 0.42 & 67 \\
\hline Dipper to nondipper $(n=37)$ & $0.50 \pm 0.18$ & $0.54 \pm 0.17$ & 0.38 & $0.04 \pm 0.18$ & -0.03 to 0.11 & 0.36 & 55 \\
\hline Nondipper to dipper $(n=31)$ & $0.54 \pm 0.17$ & $0.49 \pm 0.15$ & 0.25 & $-0.05 \pm 0.17$ & -0.12 to 0.01 & 0.34 & 49 \\
\hline \multicolumn{8}{|l|}{ Goodness of fit of AASI regression line } \\
\hline$r^{2}<0.36$ in two recordings $(n=75)$ & $0.66 \pm 0.14$ & $0.62 \pm 0.17$ & 0.06 & $-0.04 \pm 0.16$ & -0.07 to 0.00 & 0.32 & 68 \\
\hline $\begin{array}{l}r^{2} \geq 0.36 \text { in two recordings }(n=145) \\
r^{2}<0.36 \text { and } \geq 0.36(n=78)\end{array}$ & $0.40 \pm 0.13$ & $0.40 \pm 0.13$ & 0.95 & $-0.00 \pm 0.12$ & -0.02 to 0.02 & 0.24 & 44 \\
\hline$r^{2} \geq 0.36$ to $<0.36(n=36)$ & $0.43 \pm 0.15$ & $0.59 \pm 0.19$ & $<0.0001$ & $0.16 \pm 0.18$ & 0.10 to 0.22 & 0.36 & 50 \\
\hline$r^{2}<0.36$ to $\geq 0.36(n=42)$ & $0.60 \pm 0.09$ & $0.42 \pm 0.13$ & $<0.0001$ & $-0.18 \pm 0.17$ & -0.23 to -0.13 & 0.32 & 55 \\
\hline
\end{tabular}

Plus - minus values are mean $\pm \mathrm{SD}$. Absolute differences $(\Delta)$ were computed as repeat minus first recording. The repeatability coefficient (RC) is twice the $\mathrm{SD}$ of the signed differences between the duplicate measurements. RC was also expressed as a percentage of four times the SD of the mean of the paired recordings ( $\mathrm{pMV}$ ). AASI, ambulatory arterial stiffness index.

gated the reproducibility of 24-h PP from repeat ambulatory BP recordings in 133 untreated hypertensive patients. The SD of the paired differences was $4 \mathrm{mmHg}$ compared with 7 and $9 \mathrm{mmHg}$ in our Nijmegen and SystEur trial patients, respectively. In Stergiou's study, the 24-h PP averaged $( \pm \mathrm{SD}) 47.5 \pm 7.4$ and $46.4 \pm 7.9 \mathrm{mmHg}$ on first and repeat measurement. The nonreported $P$-value for the difference $(-1.1 \mathrm{mmHg})$ was therefore $0.0015\left[1.1 /\left(4 / 133^{0.5}\right)\right]$. We estimated that in Stergiou's study the repeatability coefficient expressed as a percentage of near maximal variation was approximately $27 \%$ [24], which at first sight seems somewhat smaller than in our Syst-Eur trial patients (37\%). However, in 16 of Stergiou's patient who were older than 60 years, the SD of the paired differences in the 24-h PP was $5 \mathrm{mmHg}$ [24], which would have yielded a repeatability estimate almost identical to that in our Syst-Eur trial cohort.

Gosse et al. [25] evaluated the reproducibility of QKD100-60 and AASI in 38 volunteers, who were selected from a cohort of 469 patients and who underwent repeat ambulatory recordings within 2 weeks. According to the French group [25], QKD100-60 (mean =224 ms; coefficient of variation $=4 \%$ ) had a better reproducibility than AASI ( mean $=0.60$; coefficient of variation $=25 \%$ ). Coefficient of variation is the SD of the differences between paired measurements (SDD) divided by the mean of all measurements. The coefficient of variation does not account for the possible range of biological variation in the two ambulatory measures of arterial stiffness. We, therefore, expressed repeatability (twice SDD) as a percentage of near maximal variation in the measurements as given by four times the SD. Had the French investigators [25] expressed reproducibility in this way with the application of a proper test statistic, their results and conclusions could possibly have been different.
Matsui et al. [26] studied the reproducibility of the brachial-ankle pulse wave velocity (baPWV) and the carotid augmentation index (cAIX) in 103 hypertensive patients. The interval between the repeat measurements was 4 weeks. They measured baPWV, using a volumeplethysmographic device with four cuffs fitted with oscillometric sensors, which were wrapped around the upper arms and ankles and automatically and simultaneously inflated. The measurement of cAIX was done by multielement applanation tonometry. The Japanese investigators used the correlation between paired measurements as index of agreement [26]. The correlation coefficients were 0.89 for abPWV and 0.87 for cAIX. In our study, the correlation coefficients for AASI ranged from 0.52 to 0.61 . In the absence of statistics specifically designed to assess repeatability [26], the Japanese results are difficult to interpret. Moreover, the Japanese study was done under highly standardized laboratory conditions, using a fully automated technique, whereas we measured AASI over $24 \mathrm{~h}$ during the usual daily activities of our patients.

In our current study, we found that the differences between repeat minus first AASI correlated inversely with the differences in the night-to-day ratios of diastolic BP. Two recent reports already reported on AASI's dependency on the nocturnal BP fall $[27,28]$. We also confirmed the inverse association between AASI and the night-to-day BP ratio in 1325 hypertensive patients referred to the Nijmegen clinic for ambulatory BP monitoring [29]. Several investigators demonstrated poor reproducibility of dipping status in hypertensive patients. Hernández-del Rey [30] performed repeat ambulatory monitoring on 2 consecutive days and noticed that of 611 patients $147(24.1 \%)$ participants switched from a nighttime dipper to nondipper or vice versa. Other studies [31] reported similar findings. When we stratified our study sample according to the consistency of dipping status, the repeatability coefficients expressed as a percentage of 
maximal variation were not substantially different. Thus, although there is a significant inverse association between AASI and the night-to-day BP ratio, this relation does not affect intra-individual repeatability of AASI to a large extent in stratified analyses.

In the present analysis, differences in the goodness of fit of the AASI regression line had an impact on the repeatability of AASI. Similar to recent observations by Gavish et al. [32], we demonstrated in 7604 participants enrolled in the International Database on Ambulatory BP monitoring in relation to Cardiovascular Outcomes (IDACO [33]) that the association of AASI with four main determinants of arterial stiffness (age, height, 24-h mean arterial pressure and 24-h heart rate) increased curvilinearly with the goodness of fit of the AASI regression line with most of the improvement in these associations occurring when the $r^{2}$ of the AASI regression line was 0.36 or higher. In line with these still unpublished observations, the repeatability of AASI was $68 \%$ and $44 \%$ in patients who in repeat recordings consistently had an $r^{2}$, respectively, below or above this 0.36 threshold.

The results of our study have to be interpreted in the context of its potential limitations. First, the Nijmegen cohort included $11.8 \%$ who were on antihypertensive medications. However, treatment remained unchanged between the first and repeat recording. Second, we did not standardize the ambulatory $\mathrm{BP}$ recordings across centres in terms of device type and intervals between readings. However, within patients, we used the same monitor and intervals in the first and repeat recordings. Moreover, we did not find any significant differences in repeatability between the five device types or between oscillometric versus auscultatory machines. To minimize heterogeneity, we additionally used the same programme to compute BP means and used time-weighted and robust regression.

In conclusion, estimates of group mean AASI were not different between repeat recordings. Within individual participants, the repeatability coefficients of AASI expressed as a percentage of near maximum variation were within the $50-60 \%$ range. These findings were consistent in older patients with isolated systolic hypertension and in a younger patient group with predominantly mixed hypertension. To our knowledge, this study is the first to report on the repeatability of AASI in hypertensive patients.

\section{Acknowledgements}

The authors gratefully acknowledge the expert secretarial assistance of Mrs Sandra Covens and Mrs Ya Zhu (Studies Coordinating Centre).

The European Union (grants IC15-CT98-0329-EPOGH, LSHM-CT-2006-037093 InGenious HyperCare, and
HEALTH-2007-201550 HyperGenes), the Fonds voor Wetenschappelijk Onderzoek Vlaanderen, Ministry of the Flemish Community, Brussels, Belgium (grants G.0424.03, G.0453.05 and G.0575.06), and the University of Leuven, Belgium (grants OT/99/28, OT/00/25 and OT/05/49) gave support to the Studies Coordinating Centre. J.A.S. is holder of the Pfizer Chair for Hypertension and Cardiovascular Research (http://www.kuleuven. be/mecenaat/leerstoelen/overzicht.htm) at the University of Leuven. The Dutch Heart Foundation (Dr E. Dekker grant), Den Haag, The Netherlands, supported the fellowships of D.G.D. and A.A. in Leuven.

The authors have no conflicts of interest.

\section{References}

1 Hansen TW, Staessen JA, Torp-Pedersen C, Rasmussen S, Thijs L, Ibsen $\mathrm{H}$, et al. Prognostic value of aortic pulse wave velocity as index of arterial stiffness in the general population. Circulation 2006; 113:664-670.

2 Li Y, Wang JG, Dolan E, Gao PJ, Guo HF, Nawrot T, et al. Ambulatory arterial stiffness index derived from $24-\mathrm{h}$ ambulatory blood pressure monitoring. Hypertension 2006; 47:359-364.

3 Dolan E, Thijs L, Li Y, Atkins N, McCormack P, McClory S, et al. Ambulatory arterial stiffness index as a predictor of cardiovascular mortality in the Dublin Outcome Study. Hypertension 2006; 47:365-370.

4 Leoncini G, Ratto E, Viazzi F, Vaccaro V, Parodi A, Falqui V, et al. Increased arterial stiffness index is associated with target organ damage in primary hypertension. Hypertension 2006; 48:397-403.

5 Ratto E, Leoncini G, Viazzi F, Vaccaro V, Falqui V, Parodi A, et al. Ambulatory arterial stiffness index and renal abnormalities in primary hypertension. J Hypertens 2006; 24:2033-2038.

6 Hansen TW, Staessen JA, Torp-Pedersen C, Rasmussen S, Li Y, Dolan E, et al. Ambulatory arterial stiffness index predicts stroke in a general population. J Hypertens 2006; 24:2247-2253.

7 Kikuya M, Staessen JA, Ohkubo T, Thijs L, Metoki H, Asayama K, et al. Ambulatory arterial stiffness index and 24-h ambulatory pulse pressure as predictors of mortality in Ohasama, Japan. Stroke 2007; 38:11611166.

8 Hansen TW, Li Y, Staessen JA, Jeppesen J, Rasmussen S, Wang JG, et al. Independent prognostic value of the ambulatory arterial stiffness index and aortic pulse wave velocity in a general population. J Hum Hypertens 2008; 22:214-216.

9 van der Steen MS, Pleijers AM, Lenders JW, Thien T. Influence of different supine body positions on blood pressure: consequences for night blood pressure/dipper-status. J Hypertens 2000; 18:1731-1736.

10 Staessen JA, Thijs L, Fagard R, O'Brien ET, Clement D, de Leeuw PW, et al. Predicting cardiovascular risk using conventional vs ambulatory blood pressure in older patients with systolic hypertension. J Am Med Assoc 1999; 282:539-546.

11 Mancia G, De Backer G, Dominiczak A, Cifkova R, Fagard R, Germano G, et al. 2007 Guidelines for the management of arterial hypertension. The Task Force for the management of arterial hypertension of the European Society of Hypertension (ESH) and of the European Society of Cardiology (ESC). J Hypertens 2007; 25:1105-1187.

12 Hope SL, Alun-Jones E, Sleight P. Validation of the accuracy of the Medilog ABP noninvasive blood-pressure monitor. J Ambul Monit 1988; 1:39-51.

13 Jones CR, Taylor K, Chowienczyk P, Poston L, Shennan AH. A validation of the Mobil O Graph (version 12) ambulatory blood pressure monitor. Blood Press Monit 2000; 5:233-238.

14 O'Brien E, Mee F, Atkins N, O'Malley K. Evaluation of the SpaceLabs 90202 noninvasive ambulatory recorder according to the AAMI standard and BHS criteria. J Hum Hypertens 1991; 5:223-226.

15 O'Brien E, Mee F, Atkins N, O'Malley K. Accuracy of the SpaceLabs 90207 determined by the British Hypertension Society Protocol. J Hypertens $1991 ; 9: 573-574$.

16 O'Brien E, Mee F, Atkins N, O'Malley K. Accuracy of the Takeda TM-2420/ TM-2020 determined by the British Hypertension Society Protocol. $J$ Hypertens 1991; 9:571-572.

17 Fagard R, Brguljan J, Thijs L, Staessen J. Prediction of the actual awake and asleep blood pressures by various methods of $24 \mathrm{~h}$ pressure analysis. J Hypertens 1996; 14:557-563. 
18 Li Y, Wang JG, Gao HF, Nawrot T, Wang GL, Qian YS, et al. Are published characteristics of the ambulatory blood pressure generalizable to rural Chinese ? The JingNing population study. Blood Press Monit 2005; 10:125-134.

19 Bland JM, Altman DG. Statistical methods for assessing agreement between two methods of clinical measurement. Lancet 1986; 2:307-310.

20 Dewitte K, Fierens C, Stockl D, Thienpont LM. Application of the BlandAltman plot for interpretation of method-comparison studies: a critical investigation of its practice. Clin Chem 2002; 48:799-801.

21 Staessen J, Bulpitt CJ, O'Brien E, Cox J, Fagard R, Stanton A, et al. The diurnal blood pressure profile. A population study. Am J Hypertens 1992; 5:386-392.

22 Kleinbaum DG, Kupper LL, Muller KE. Applied regression analysis and other multivariate methods, 2nd ed. Boston, Massachusetts: PWS-Kent Publishing Company; 1988.

23 Thijs L, Staessen J, Fagard R, Zachariah P, Amery A. Number of measurements required for the analysis of diurnal blood pressure profile. J Hum Hypertens 1994; 8:239-244.

24 Stergiou GS, Efstathiou SP, Argyraki CK, Gantzarou AP, Roussias LG, Mountokalakis TD. Clinic, home and ambulatory pulse pressure: comparison and reproducibility. J Hypertens 2002; 20:1987-1993.

25 Gosse P, Papaioanou G, Coulon P, Reuter S, Lemetayer P, Safar M. Can ambulatory blood-pressure monitoring provide reliable indices of arterial stiffness? Am J Hypertens 2007; 20:831-838.

26 Matsui Y, Kario K, Ishikawa J, Eguchi K, Hoshide S, Shimada K. Reproducibility of arterial stiffness indices (pulse wave velocity and augmentation index) simultaneously assessed by automated pulse wave analysis and their associated risk factors in essential hypertensive patients. Hypertens Res 2004; 27:851-857.

27 Schillaci G, Parati G, Pirro M, Pucci G, Mannarino MR, Sperandini L, et al. Ambulatory arterial stiffness index is not a specific marker of reduced arterial compliance. Hypertension 2007; 49:986-991.

28 Adiyaman A, Boggia J, Li Y, Wang JG, O'Brien E, Richart T, et al. Dipping deeper into the ambulatory arterial stiffness index. Hypertension 2007; 50:e59-e60.

29 Dechering DG, Adiyaman A, van der Steen M, Thien T. Interstudy variability in the ambulatory arterial stiffness index. Hypertension 2007; 50:e65.

30 Hernández-del Rey R, Martin-Baranera M, Sobrino J, Gorostidi M, Vinyoles $\mathrm{E}$, Sierra C, et al. Reproducibility of the circadian blood pressure pattern in 24-h versus 48-h recordings: the Spanish Ambulatory Blood Pressure Monitoring Registry. J Hypertens 2007; 25:2406-2412.

31 Cuspidi C, Meani S, Valerio C, Sala C, Fusi V, Masaidi M, et al. Reproducibility of dipping/nondipping pattern in untreated essential hypertensive patients: impact of sex and age. Blood Press Monit 2007; 12:101-106.

32 Gavish B, Ben-Dov IZ, Bursztyn M. Linear relationship between systolic and diastolic blood pressure monitored over $24 \mathrm{~h}$ : assessment and correlates. J Hypertens 2008; 26:199-208.

33 Thijs L, Hansen TW, Kikuya M, Björklund-Bodegard K, Li Y, Dolan E, et al. The International Database of Ambulatory blood pressure in relation to Cardiovascular Outcome (IDACO): protocol and research perspectives. Blood Press Monit 2007; 12:255-262. 